\title{
CONSERVATION LAWS FOR EQUATIONS RELATED TO SOIL WATER EQUATIONS
}

\author{
C. M. KHALIQUE AND F. M. MAHOMED \\ Received 10 April 2003 and in revised form 7 May 2004
}

We obtain all nontrivial conservation laws for a class of $(2+1)$ nonlinear evolution partial differential equations which are related to the soil water equations. It is also pointed out that nontrivial conservation laws exist for certain classes of equations which admit point symmetries. Moreover, we associate symmetries with conservation laws for special classes of these equations.

\section{Introduction and theory}

A mathematical model was developed to simulate soil water infiltration, redistribution, and extraction in a bedded soil profile overlaying a shallow water table and irrigated by a line source drip irrigation system (see $[19,20]$ ). The governing partial differential equation can be written as

$$
C(\psi) \psi_{t}=\left(K(\psi) \psi_{x}\right)_{x}+\left(K(\psi)\left(\psi_{z}-1\right)\right)_{z}-S(\psi),
$$

where $\psi$ is soil moisture pressure head, $C(\psi)$ is specific water capacity, $K(\psi)$ is unsaturated hydraulic conductivity, $S(\psi)$ is a sink or source term, $t$ is time, $x$ is the horizontal axis, and $z$ is the vertical axis which is considered positive downward.

Equation (1.1) is an approximation within the context of the theory of mixtures. It is studied here in the form (1.1) as a first step. An introduction to the general theory of mixtures can be found in the book by Rajagopal and Tao [17] which contains references to the earlier papers of Truesdell that have had a great impact on the development of the theory. This approach is rather general and takes into account a variety of physical mechanisms and chemical reactions. Also of crucial importance within such a theory is the choice of boundary conditions and the problems associated with the choice of these conditions (see [6]). The book also provides numerous references on flow through porous media and the interested reader can consult the relevant references therein.

Group classification of (1.1) with respect to admitted point transformation groups was done in [2]. Some exact solutions were also obtained in the paper just cited. 
Equation (1.1) can be rewritten as

$$
u_{t}=\left(k(u) u_{x}\right)_{x}+\left(k(u) u_{z}\right)_{z}+l(u) u_{z}+p(u)
$$

where

$$
u=\int C(\psi) d \psi, \quad k(u)=\frac{K(\psi)}{C(\psi)}, \quad l(u)=\frac{-K^{\prime}(\psi)}{C(\psi)}, \quad p(u)=-S(\psi) .
$$

Equation (1.2) has received a fair amount of attention recently. Group classification of (1.2) with respect to admitted point transformation groups was performed in [1]. Classification of (1.2) in the case when $l(u)=0$ with respect to Lie point symmetries was given in $[3,4]$ (see also [5] and [18, Section 10.7]).

Special cases of (1.1) and (1.2) were considered for conservation laws and associated symmetries in [10].

In this paper, we obtain all nontrivial conservation laws for (1.2). We list all the classes of (1.2) which admit point symmetries and for which nontrivial conservation laws exist. Moreover, for special classes of such equations we associate symmetries with conservation laws. Finally we act, by use of a symmetry, on a known conservation law to produce another conservation law.

We first briefly present the notation and pertinent results used in this paper. The summation convention is used where appropriate.

Consider a $k$ th-order $(k \geq 1)$ system of differential equations of $n$ independent variables and $m$ dependent variables

$$
E^{\beta}\left(x, u, u_{(1)}, \ldots, u_{(k)}\right)=0, \quad \beta=1, \ldots, m,
$$

where $u_{(p)}$ denotes the various collections of $p$ th-order partial derivatives. The maximal order of the equations that occur in (1.4) is $k$.

A conservation law for system (1.4) is a relation

$$
D_{i} T^{i}=0
$$

where $T^{i}=T^{i}\left(x, u, u_{(1)}, \ldots, u_{(k-1)}\right)$ are differential functions, which is satisfied on the solutions of (1.4). Here $D_{i}=\partial / \partial x^{i}+u_{i}^{\alpha} \partial / \partial u^{\alpha}+\cdots$ is the total differentiation operator with respect to $x^{i}$.

A Lie point symmetry generator has the form (see, e.g., the books $[8,15,16]$ for an account on the symmetry approach to differential equations)

$$
X=\xi^{i} \frac{\partial}{\partial x^{i}}+\eta^{\alpha} \frac{\partial}{\partial u^{\alpha}}+\zeta_{i}^{\alpha} \frac{\partial}{\partial u_{i}^{\alpha}}+\zeta_{i_{1} i_{2}}^{\alpha} \frac{\partial}{\partial u_{i_{1} i_{2}}^{\alpha}}+\cdots,
$$

where the $\xi^{i}(i=1, \ldots, n)$ and $\eta^{\alpha}(\alpha=1, \ldots, m)$ are functions of the independent and dependent variables, and the additional coefficients are given by

$$
\begin{gathered}
\zeta_{i}^{\alpha}=D_{i}\left(\eta^{\alpha}\right)-u_{j}^{\alpha} D_{i}\left(\xi^{j}\right), \\
\zeta_{i_{1} \cdots i_{s}}^{\alpha}=D_{i_{s}}\left(\zeta_{i_{1} \cdots i_{s-1}}^{\alpha}\right)-u_{j i_{1} \cdots i_{s-1}}^{\alpha} D_{i_{s}}\left(\xi^{j}\right), \quad s>1 .
\end{gathered}
$$


It is well known that Noether's theorem [14] provides a constructive way for finding conservation laws for Euler-Lagrange differential equations once their symmetries are known. In the absence of a Lagrangian for a differential equation one can resort to the direct method of constructing conservation laws via (1.5). A symmetry condition sometimes helps in fixing some of the arbitrary elements in the differential equation.

A Lie point symmetry generator $X$ admitted by system (1.4) is said to be associated with a conservation law with conserved vector $T=\left(T^{1}, \ldots, T^{n}\right)$ of system (1.4) if the relations [11]

$$
X\left(T^{i}\right)+T^{i} D_{j}\left(\xi^{j}\right)-T^{j} D_{j}\left(\xi^{i}\right)=0, \quad i=1, \ldots, n,
$$

hold. This also applies for Noether symmetries [9].

It has been shown in [12] that if $X$ is any point symmetry generator of (1.4) and $T^{i}$, $i=1, \ldots, n$, are the components of a conserved vector of (1.4), then

$$
T_{*}^{i}=X\left(T^{i}\right)+T^{i} D_{j}\left(\xi^{j}\right)-T^{j} D_{j}\left(\xi^{i}\right), \quad i=1, \ldots, n,
$$

are components of a conserved vector of (1.4). If in addition $[X, Y]=Z$ (see [12]), where $Y$ is associated with the conserved vector $T$ with components $T^{i}$ and $X$ is admitted by (1.4), then $T_{*}$ defined by (1.9) is trivial if $Z=b Y$ for $b$ a constant.

\section{Construction of conservation laws for (1.2)}

In this section, we construct conservation laws for (1.2), that is, we invoke (1.5), namely,

$$
D_{t} T^{1}+D_{x} T^{2}+D_{z} T^{3}=0
$$

on the solutions of (1.2). We cannot use Noether's theorem [14] here as there is no Lagrangian for (1.2).

The separation of the second-order partial derivatives of $u$ in the determining equation of (2.1) results in the following system of equations:

$$
\begin{gathered}
u_{t t}: \frac{\partial T^{1}}{\partial u_{t}}=0, \\
u_{x t}: \frac{\partial T^{1}}{\partial u_{x}}+\frac{\partial T^{2}}{\partial u_{t}}=0, \\
u_{z t}: \frac{\partial T^{1}}{\partial u_{z}}+\frac{\partial T^{3}}{\partial u_{t}}=0, \\
u_{z x}: \frac{\partial T^{2}}{\partial u_{z}}+\frac{\partial T^{3}}{\partial u_{x}}=0,
\end{gathered}
$$




$$
\begin{aligned}
& u_{x x}: k(u) \frac{\partial T^{1}}{\partial u}+\frac{\partial T^{2}}{\partial u_{x}}=0, \\
& u_{z z}: k(u) \frac{\partial T^{1}}{\partial u}+\frac{\partial T^{3}}{\partial u_{z}}=0
\end{aligned}
$$

rest : $\frac{\partial T^{1}}{\partial t}+\frac{\partial T^{1}}{\partial u}\left(k^{\prime} u_{x}^{2}+k^{\prime} u_{z}^{2}+l(u) u_{z}+p(u)\right)+\frac{\partial T^{2}}{\partial x}+\frac{\partial T^{2}}{\partial u} u_{x}+\frac{\partial T^{3}}{\partial z}+\frac{\partial T^{3}}{\partial u} u_{z}=0$.

The solution of this system (2) yields the conserved vector components

$$
\begin{aligned}
& T^{1}=-a(t, x, z) u_{x}+b(t, x, z) u_{z}+A(t, x, z) u+B(t, x, z), \\
& T^{2}=a(t, x, z) u_{t}-k(u) u_{x} A(t, x, z)-c_{1}(t, x, z, u) u_{z}+d(t, x, z, u), \\
& T^{3}=-b(t, x, z) u_{t}-k(u) u_{z} A(t, x, z)+c_{1}(t, x, z, u) u_{x}+c_{2}(t, x, z, u),
\end{aligned}
$$

where the functions $a$ to $B$ satisfy

$$
\begin{gathered}
\frac{\partial a}{\partial x}-\frac{\partial b}{\partial z}=0 \\
\frac{\partial a}{\partial t}+k(u) \frac{\partial A}{\partial x}-\frac{\partial d}{\partial u}-\frac{\partial c_{1}}{\partial z}=0 \\
\frac{\partial b}{\partial t}+l(u) A-\frac{\partial c_{1}}{\partial x}-k(u) \frac{\partial A}{\partial z}+\frac{\partial c_{2}}{\partial u}=0 \\
\frac{\partial A}{\partial t} u+\frac{\partial B}{\partial t}+p(u) A+\frac{\partial d}{\partial x}+\frac{\partial c_{2}}{\partial z}=0
\end{gathered}
$$

From equations (2.4) it follows that

$$
k(u)\left(\frac{\partial^{2} A}{\partial x^{2}}+\frac{\partial^{2} A}{\partial z^{2}}\right)-l(u) \frac{\partial A}{\partial z}+p^{\prime}(u) A+\frac{\partial A}{\partial t}=0 .
$$

The use of (2.5) enables us to classify all cases for which conservation laws for (1.2) exist. We find the following cases.

(1) For arbitrary $k(u), l(u)$, and $p(u)$ we get trivial conservation laws since $A=0$.

Nontrivial conservation laws are obtained in the following cases.

(2) $p=p_{0}+p_{1} u, l=l_{0}, k=k_{0} \neq 0$, where $p_{0}, p_{1}, l_{0}$, and $k_{0}$ are arbitrary constants. The function $A$ satisfies

$$
\left(A_{x x}+A_{z z}\right) k_{0}-l_{0} A_{z}+p_{1} A+A_{t}=0
$$

together with system (2.4).

(3) $p=p_{0}+p_{1} u, l=l_{0}, k=k(u)$ with $k^{\prime}(u) \neq 0$, where $p_{0}, p_{1}$, and $l_{0}$ are arbitrary constants and

$$
A=\exp \left(-p_{1} t\right) f\left(x, z+l_{0} t\right)
$$

where $f$ solves $f_{x x}+f_{\tau \tau}=0, \tau=z+l_{0} t$. System (2.4) also must be satisfied. 
(4) $p=p_{0}+p_{1} u, l=l(u)$ with $l^{\prime}(u) \neq 0, k=N l(u)+M$, where $p_{0}, p_{1}, N$, and $M$ are arbitrary constants and

$$
A=\exp \left(-p_{1} t\right) f(x, N z-M t)
$$

where $f$ solves $f_{x x}+N^{2} f_{\tau \tau}-f_{\tau}=0, \tau=N z-M t$. System (2.4) needs to be satisfied as well.

(5) $p=p_{0}+p_{1} u, l=l(u)$ with $l^{\prime}(u) \neq 0, k=k(u)$ with $k(u) \neq N l(u)+M$, where $p_{0}$, $p_{1}, N$, and $M$ are arbitrary constants, and

$$
A=f_{0} x \exp \left(-p_{1} t\right)+g_{0} \exp \left(-p_{1} t\right)
$$

in which $f_{0}$ and $g_{0}$ are constants. In addition $A$ must satisfy (2.4).

(6) $p=p(u)$ with $p^{\prime \prime}(u) \neq 0, l(u)=N p^{\prime}(u)+M$, and $k(u)=R p^{\prime}(u)+S$, where $N, M$, $R$, and $S$ are arbitrary constants. We have two cases, namely,

$$
\begin{gathered}
A=\exp \left(\frac{S t}{R}\right) f(x,(S N-R M) t-R z), \quad R \neq 0, \\
R f_{x x}+R^{3} f_{\tau \tau}+N R f_{\tau}+f=0, \quad \tau=(S N-R M) t-R z, \\
A=\exp \left(\frac{z}{N}\right) f(t, x), \quad N \neq 0, R=0, \\
S N^{2} f_{x x}+(S-M N) f+N^{2} f_{t}=0 .
\end{gathered}
$$

In both cases $A$ is further constrained by system (2.4).

(7) $p=p(u)$ with $p^{\prime \prime}(u) \neq 0, l(u)=N p^{\prime}(u)+M, k=k(u)$ with $k(u) \neq R p^{\prime}(u)+S$, where $N, M, R$, and $S$ are arbitrary constants. Here $A$ is given by

$$
A=\exp \left(\frac{z+M t}{N}\right)\left[f_{0} \cos \left(\frac{x}{N}\right)+g_{0} \sin \left(\frac{x}{N}\right)\right], \quad N \neq 0,
$$

where $f_{0}$ and $g_{0}$ are constants. These values of $A$ are constrained by (2.4).

(8) $p=p(u)$ with $p^{\prime \prime}(u) \neq 0, l=l(u)$ with $l(u) \neq N p^{\prime}(u)+M, k(u)=L l(u)+Q p^{\prime}(u)+$ $R$, where $L, Q, R, M$, and $N$ are arbitrary constants. The function $A$ has the value

$$
A=f(x) \exp \left(\frac{R t-L z}{Q}\right), \quad Q \neq 0,
$$

where $f$ solves $f^{\prime \prime}+f\left(L^{2}+Q\right) / Q^{2}=0$. Again $A$ must satisfy (2.4).

In the above, only the cases (5), (7), and (8) give rise to two nontrivial conservation laws each. The other cases each result in an infinite number of nontrivial conservation laws. We illustrate this point by considering the following two examples. 
For case (3), the solution of system (2.4) with the choices $a=b=c_{1}=B=0$ yields

$$
\begin{gathered}
T^{1}=f\left(x, z+l_{0} t\right) u \exp \left(-p_{1} t\right), \\
T^{2}=-k(u) u_{x} \exp \left(-p_{1} t\right) f\left(x, z+l_{0} t\right)+\exp \left(-p_{1} t\right) f_{x} \int_{0}^{u} k\left(u^{\prime}\right) d u^{\prime}, \\
T^{3}=-k(u) u_{z} \exp \left(-p_{1} t\right) f\left(x, z+l_{0} t\right)+\exp \left(-p_{1} t\right) f_{\tau} \int_{0}^{u} k\left(u^{\prime}\right) d u^{\prime}-l_{0} u f \exp \left(-p_{1} t\right),
\end{gathered}
$$

where $f$ satisfies $f_{x x}+f_{\tau \tau}=0, \tau=z+l_{0} t$. Equations (2.14) give rise to an infinite number of conserved vectors.

Similarly for case (5) with the choices $a=b=c_{1}=B=0$, we obtain

$$
\begin{aligned}
T^{1}= & {\left[f_{0} x \exp \left(-p_{1} t\right)+g_{0} \exp \left(-p_{1} t\right)\right] u, } \\
T^{2}= & -k(u) u_{x}\left[f_{0} x \exp \left(-p_{1} t\right)+g_{0} \exp \left(-p_{1} t\right)\right] \\
& +f_{0} \exp \left(-p_{1} t\right) \int_{0}^{u} k\left(u^{\prime}\right) d u^{\prime}+\alpha(t, x, z), \\
T^{3}= & -k(u) u_{z}\left[f_{0} x \exp \left(-p_{1} t\right)+g_{0} \exp \left(-p_{1} t\right)\right] \\
& -\left[f_{0} x \exp \left(-p_{1} t\right)+g_{0} \exp \left(-p_{1} t\right)\right] \int_{0}^{u} l\left(u^{\prime}\right) d u^{\prime}+\beta(t, x, z),
\end{aligned}
$$

where $\alpha$ and $\beta$ are constrained by

$$
p_{0}\left[f_{0} x \exp \left(-p_{1} t\right)+g_{0} \exp \left(-p_{1} t\right)\right]+\alpha_{x}+\beta_{z}=0
$$

The components (2.15) result in two conserved vectors.

The conservation laws for the other cases can be constructed in a similar fashion.

The only classes in the symmetry classification (see [7]) which have nontrivial conservation laws are (the notation used in the following corresponds to that of [7])

(I.2) $k(u)$ arbitrary, $p(u)=0, l(u)=0$,

(II) $k(u)=e^{u}$,

(1) $l(u)=A e^{u}, p(u)=B e^{u}+C(A, B$, and $C$ are arbitrary constants, $A \neq 0)$,

(4) $l(u)=0$, (i) $p= \pm e^{u}+\delta, \delta= \pm 1$, (iii) $p(u)=\delta, \delta \pm 1$, (iv) $p(u)=0$,

(III) $k(u)=u^{\sigma}, \sigma \neq 0,-1$,

(1) $l(u)=A u^{\sigma}, p(u)=B u^{\sigma+1}-(C / \sigma) u(A, B, C$, and $\sigma$ are constants, $A \neq 0)$,

(2) $l(u)=C u^{\mu}, p(u)=A u^{1+2 \mu-\sigma}, \mu=2 \sigma$,

(3) $l(u)=0$, (i) $p(u)= \pm u^{\nu}, v \neq 0,1, v=\sigma+1$, (ii) $p(u)= \pm u^{\sigma+1}+\delta u, \delta= \pm 1$, $\sigma=$ const, (iii) $p(u)=\delta u, \delta \pm 1$, (iv) $p(u)=0$,

(IV) $k(u)=u^{-1}, l(u)=0$, (ii) $p(u)=\delta u \pm 1, \delta= \pm 1$ (iii) $p(u)= \pm 1$, (iv) $p(u)=\delta u$, $\delta= \pm 1,(\mathrm{v}) p(u)=0$,

(V) $k(u)=1$,

(3) $l(u)=A \ln u, p(u)=u(B \ln u+C)$,

(6) $l(u)=A u, p(u)=B u+C$,

(8) $l(u)=0$, (i) $p(u)=\delta u, \delta= \pm 1$, (ii) $p(u)= \pm 1$, (vi) $p(u)=0$. 


\section{Symmetries associated with conservation laws}

In this section, we give two examples of symmetries associated with conservation laws. Firstly, we consider the case III.3.(ii) of [7], namely, $k(u)=u^{\sigma}, \sigma \neq 0,-1 ; p(u)=\delta u^{\sigma+1}+$ $\delta u, \delta= \pm 1, \sigma=$ constant, $l(u)=0$.

The point symmetries for this case are (see [7])

$$
\begin{gathered}
X_{1}=\frac{\partial}{\partial x}, \quad X_{2}=\frac{\partial}{\partial z}, \quad X_{3}=\frac{\partial}{\partial t}, \\
X_{4}=z \frac{\partial}{\partial x}-x \frac{\partial}{\partial z}, \\
X_{5}=\exp (-\delta \sigma t)\left(\frac{\partial}{\partial t}+\delta u \frac{\partial}{\partial u}\right) .
\end{gathered}
$$

We invoke relations (1.8) in order to associate the symmetries (3.1) with the conservation laws given in case (6) for the appropriate values of $k, p$, and $l$.

We list the conservation laws associated with the above symmetries.

(1) For $X_{1}$ we have the associated conserved components by use of (1.8), that is, $X_{1}\left(T^{i}\right)=0, i=1,2,3$. They are (by the choices $a=b=d=c_{1}=0$ and $B=0$ )

$$
\begin{gathered}
T^{1}=f(z) u \exp (-\delta t), \\
T^{2}=-u^{\sigma} u_{x} f(z) \exp (-\delta t), \\
T^{3}=-u^{\sigma} u_{z} f(z) \exp (-\delta t)+\frac{1}{1+\sigma} u^{1+\sigma} f^{\prime}(z) \exp (-\delta t),
\end{gathered}
$$

where

$$
f^{\prime \prime}+\delta(1+\sigma) f=0
$$

(2) For $X_{2}$ we have the associated conserved components (here $X_{2}\left(T^{i}\right)=0, i=1,2,3$ ) (the choices are $a=b=c_{1}=c_{2}=0$ and $B=0$ )

$$
\begin{gathered}
T^{1}=f(x) u \exp (-\delta t), \\
T^{2}=-u^{\sigma} u_{x} f(x) \exp (-\delta t)+\frac{1}{1+\sigma} u^{1+\sigma} f^{\prime}(x) \exp (-\delta t), \\
T^{3}=-u^{\sigma} u_{z} f(x) \exp (-\delta t),
\end{gathered}
$$

where $f$ satisfies (3.3).

(3) For $X_{3}$ there is no associated nontrivial conservation law.

(4) For $X_{4}$, the use of the relations (1.8), namely, $X_{4}^{[1]} T^{1}=0, X_{4}^{[1]} T^{2}-T^{3}=0$, and $X_{4}^{[1]} T^{3}+T^{2}=0$, after the simple choices $a=b=c_{1}=0$ and $B=0$, results in the associated conserved components

$$
\begin{gathered}
T^{1}=f(\tau) u \exp (-\delta t), \\
T^{2}=-u^{\sigma} u_{x} f(\tau) \exp (-\delta t)+\frac{2 x}{1+\sigma} u^{1+\sigma} f^{\prime}(\tau) \exp (-\delta t), \\
T^{3}=-u^{\sigma} u_{z} f(\tau) \exp (-\delta t)+\frac{2 z}{1+\sigma} u^{1+\sigma} f^{\prime}(\tau) \exp (-\delta t),
\end{gathered}
$$


where

$$
4 \tau f^{\prime \prime}+4 f^{\prime}+\delta(1+\sigma) f=0, \quad \tau=x^{2}+z^{2} .
$$

(5) For $X_{5}$ there is no associated nontrivial conservation law.

We now act, using (1.9), with a symmetry on a known conservation law to produce another conservation law.

If one acts with $X_{1}$ on the conservation law associated with $X_{4}$, we obtain another conservation law since $\left[X_{1}, X_{4}\right]=-X_{2}[12]$, namely, the generated conserved components are

$$
\begin{gathered}
T_{*}^{1}=2 x f^{\prime}(\tau) u \exp (-\delta t), \\
T_{*}^{2}=-2 x u^{\sigma} u_{x} f^{\prime}(\tau) \exp (-\delta t)+\frac{2}{1+\sigma} u^{1+\sigma} f^{\prime}(\tau) \exp (-\delta t)+\frac{4 x^{2}}{1+\sigma} u^{1+\sigma} f^{\prime \prime}(\tau) \exp (-\delta t), \\
T_{*}^{3}=-2 x u^{\sigma} u_{z} f^{\prime}(\tau) \exp (-\delta t)+\frac{4 x z}{1+\sigma} u^{1+\sigma} f^{\prime \prime}(\tau) \exp (-\delta t),
\end{gathered}
$$

where $f$ satisfies (3.6). One can further act with $X_{1}$ on the above components to obtain another conserved vector. In this manner one can generate an infinite number of conservation laws by repeated action of $X_{1}$.

If one now acts with $X_{2}$ on the conservation law associated with $X_{4}$, we obtain another conservation law since $\left[X_{2}, X_{4}\right]=X_{1}[12]$ :

$$
\begin{gathered}
T_{\dagger}^{1}=2 z f^{\prime}(\tau) u \exp (-\delta t), \\
T_{\dagger}^{2}=-2 z u^{\sigma} u_{x} f^{\prime}(\tau) \exp (-\delta t)+\frac{4 x z}{1+\sigma} u^{1+\sigma} f^{\prime \prime}(\tau) \exp (-\delta t), \\
T_{\dagger}^{3}=-2 z u^{\sigma} u_{z} f^{\prime}(\tau) \exp (-\delta t)+\frac{2}{1+\sigma} u^{1+\sigma} f^{\prime}(\tau) \exp (-\delta t)+\frac{4 z^{2}}{1+\sigma} u^{1+\sigma} f^{\prime \prime}(\tau) \exp (-\delta t),
\end{gathered}
$$

where $f$ satisfies (3.6). Again one can further act with $X_{2}$ on these components to obtain another conserved vector. In this way one can generate an infinite number of conservation laws via repeated action of $X_{2}$.

Note that these conservation laws may be associated with a linear combination of the five symmetries of the equation.

We next consider the case $k(u)=\delta(1+\sigma) u^{\sigma}+\delta, p(u)=\delta u^{\sigma+1}+\delta u, \delta= \pm 1, \sigma=$ constant, $l(u)=0$.

The point symmetries admitted by the equation for this case are [7]

$$
X_{1}=\frac{\partial}{\partial x}, \quad X_{2}=\frac{\partial}{\partial z}, \quad X_{3}=\frac{\partial}{\partial t} .
$$

We invoke relations (1.8) in order to associate the symmetries (3.1) with the conservation laws given in case (6) for the appropriate values of $k, p$, and $l$.

We deduce the conservation laws associated with each of the symmetries (3.9). 
For $X_{3}$ we have (by the choices $a=b=c_{1}=0$ and $B=0$ ) the associated conserved components

$$
\begin{gathered}
T^{1}=A u, \\
T^{2}=-\left[\delta(\sigma+1) u^{\sigma}+\delta\right] u_{x} A+\delta u^{1+\sigma} A_{x}+\delta u A_{x} \\
T^{3}=-\left[\delta(\sigma+1) u^{\sigma}+\delta\right] u_{z} A+\delta u^{1+\sigma} A_{z}+\delta u A_{z}
\end{gathered}
$$

where $A$ satisfies

$$
A_{t}=0, \quad A_{x x}+A_{z z}+A=0
$$

The conserved components (3.10) have associated symmetry $X_{1}$ if $A_{x}=0$ and $X_{2}$ provided $A_{z}=0$.

\section{Concluding remarks}

We have obtained all nontrivial conservation laws for (1.2) which have been extensively studied in the literature for various other properties such as symmetries and exact solutions. It has been shown that for arbitrary elements these equations possess trivial conservation laws. There are seven cases that arise for which this class of $(2+1)$ evolution equations have nontrivial conserved vectors. Among these, three cases result in each admitting two nontrivial conserved vectors. Each of the other cases yields an infinite number of nontrivial conservation laws. Notwithstanding, we have also provided all the classes in the symmetry classification which have nontrivial conserved vectors. Moreover, we have associated symmetries to conservation laws for special classes of these equations. The symmetry condition enables one to further constrain the remaining arbitrary elements that are contained in system (2.4) and also relates symmetry to the conserved vectors.

Further work can be done on the reduction and solutions of (1.2) which admit symmetries that preserve the conservation laws. In this regard, the authors of [13] have used this invariance of a conservation law related to volume to obtain solutions for a problem in thin films.

\section{Acknowledgment}

We thank the referees for providing us with further references.

\section{References}

[1] V. A. Baikov, R. K. Gazizov, N. H. Ibragimov, and V. F. Kovalev, Group analysis of soil water equations, CRC Handbook of Lie Group Analysis of Differential Equations. Vol.2, Applications in Engineering and Physical Sciences, (N. H. Ibragimov, ed.), CRC Press, Folrida, 1995, pp. 228-237.

[2] Water redistribution in irrigated soil profiles: invariant solutions of the governing equation, Nonlinear Dynam. 13 (1997), no. 4, 395-409.

[3] V. A. Dorodnitsyn, I. V. Knyazeva, and S. R. Svirshchevski1̌, Group properties of the anisotropic heat equation with source $T_{t}=\sum_{i}\left(K_{i}(T) T_{x_{i}}\right)_{x_{i}}+Q(T)$, preprint 134, 1982, Keldysh institute of Applied Mathematics, Russian Academy of Sciences, Moscow. 
[4] Group properties of the heat equation with source in the two-dimensional and threedimensional cases, Differentsial'nye Uravneniya 19 (1983), no. 7, 1215-1223 (Russian).

[5] V. A. Galaktionov, V. A. Dorodnitsyn, G. G. Elenin, S. P. Kurdyumov, and A. A. Samarskii, A quasilinear heat equation with a source: peaking, localization, symmetry, exact solutions, asymtotics, structures, Itogi Nauki i Tekhniki, Seriya Sovremennye Problemy Matematiki. Noveishie Dostizheniya, vol. 28, VINITI, Moscow, 1986, English translation in J. Sovient Math. 41 (1988) 1222-1292.

[6] M. Gandhi, K. R. Rajagopal, and A. S. Wineman, A universal relation in torsion for a mixture of solid and fluid, J. Elasticity 15 (1985), no. 2, 155-165.

[7] R. K. Gazizov, Earth sciences, CRC Handbook of Lie Group Analysis of Differential Equations. Vol. 2 (N. H. Ibragimov, ed.), CRC Press, 1995.

[8] N. H. Ibragimov, Transformation Groups Applied to Mathematical Physics, Mathematics and Its Applications (Soviet Series), D. Reidel Publishing, Dordrecht, 1985.

[9] N. H. Ibragimov, A. H. Kara, and F. M. Mahomed, Lie-Bäcklund and Noether symmetries with applications, Nonlinear Dynam. 15 (1998), no. 2, 115-136.

[10] A. H. Kara and C. M. Khalique, Conservation laws and associated symmetries for some classes of soil water motion equations, Internat. J. Non-Linear Mech. 36 (2001), no. 7, 1041-1045.

[11] A. H. Kara and F. M. Mahomed, Relationship between symmetries and conservation laws, Internat. J. Theoret. Phys. 39 (2000), no. 1, 23-40.

[12] A basis of conservation laws for partial differential equations, J. Nonlinear Math. Phys. 9 (2002), no. suppl. 2, 60-72.

[13] E. Momoniat, D. P. Mason, and F. M. Mahomed, Non-linear diffusion of an axisymmetric thin liquid drop: group-invariant solution and conservation law, Internat. J. Non-Linear Mech. 36 (2001), no. 6, 879-885.

[14] E. Noether, Invariante Variationsprobleme, Nachr. v. d. Ges. d. Wiss. zu Göttingen 2 (1918), 235-257 (German).

[15] P. J. Olver, Applications of Lie Groups to Differential Equations, Graduate Texts in Mathematics, vol. 107, Springer-Verlag, New York, 1986.

[16] L. V. Ovsiannikov, Group Analysis of Differential Equations, Academic Press, New York, 1982, translation edited by W. F. Ames.

[17] K. R. Rajagopal and L. Tao, Mechanics of Mixtures, Series on Advances in Mathematics for Applied Sciences, vol. 35, World Scientific Publishing, New Jersey, 1995.

[18] S. R. Svirshchevskiü, Evolution equations. I: diffusion equations, CRC Handbook of Lie Group Analysis of Differential Equations. Vol. 1 (N. H. Ibragimov, ed.), CRC Press, 1994.

[19] G. Vellidis and A. G. Smajstrla, Modelling of soil water redistribution and extraction patterns of drip-irrigated tomatoes above a shallow water table, Trans. Amer. Soc. Agric. Engin. 35 (1992), no. 1, 183-191.

[20] G. Vellidis, A. G. Smajstrla, and F. S. Zazueta, Soil water redistribution and extraction patterns of drip-irrigated tomatoes above a shallow water table, Trans. Amer. Soc. Agric. Engin. 33 (1990), no. 5, 1525-1530.

C. M. Khalique: International Institute for Symmetry Analysis and Mathematical Modelling and Department of Mathematical Sciences, North West University, Private Bag X2046, Mmabatho 2735, South Africa

E-mail address: khaliquecm@uniwest.ac.za

F. M. Mahomed: Centre for Differential Equations, Continuum Mechanics and Applications, School of Computational and Applied Mathematics, University of the Witwatersrand, Private Bag 3, Wits 2050, Johannesburg, South Africa

E-mail address: fmahomed@cam.wits.ac.za 


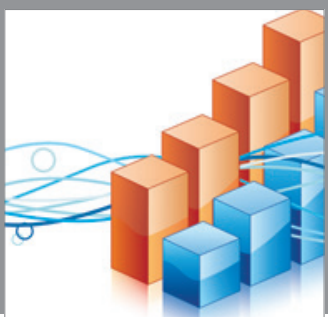

Advances in

Operations Research

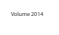

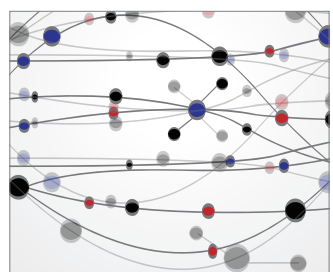

\section{The Scientific} World Journal
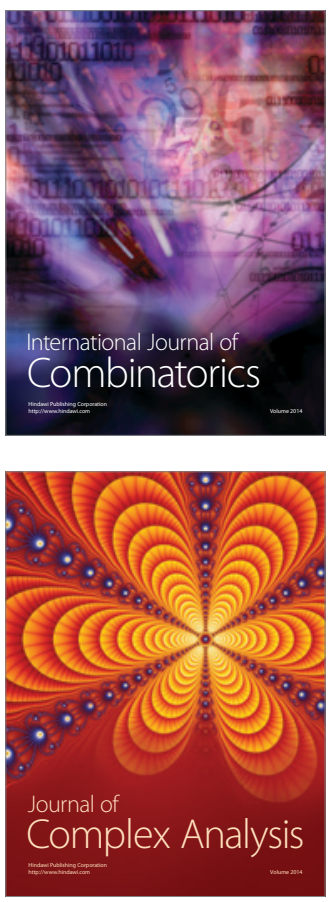

International Journal of

Mathematics and

Mathematical

Sciences
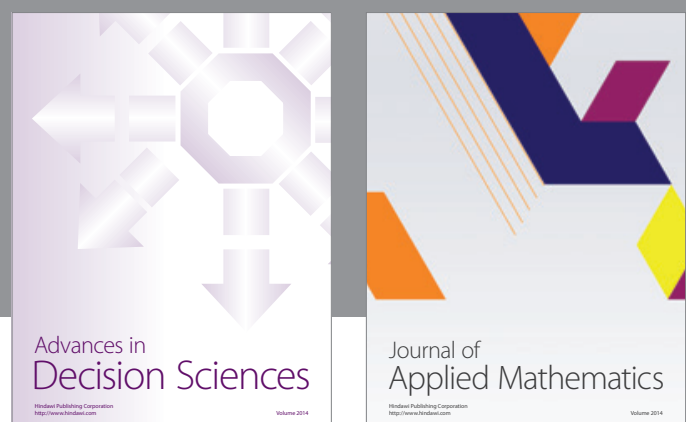

Journal of

Applied Mathematics
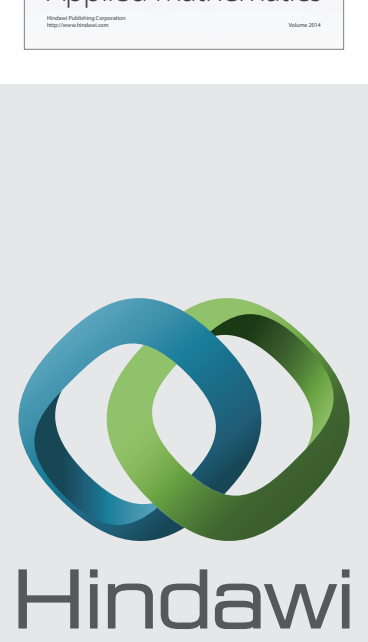

Submit your manuscripts at http://www.hindawi.com
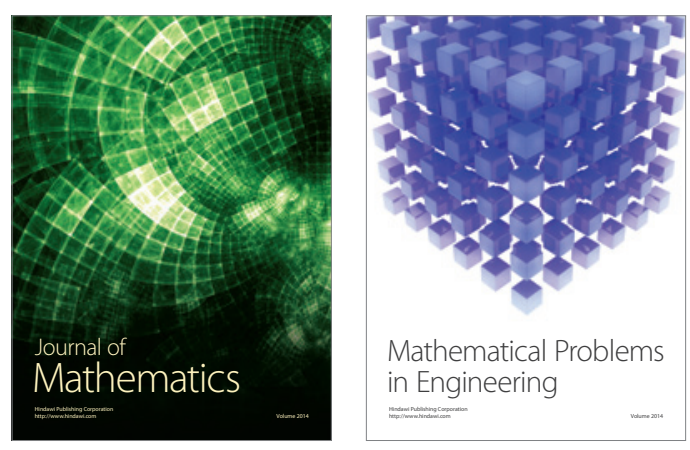

Mathematical Problems in Engineering
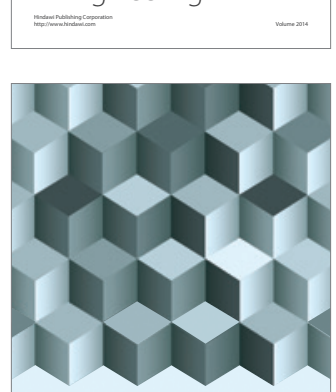

Journal of

Function Spaces
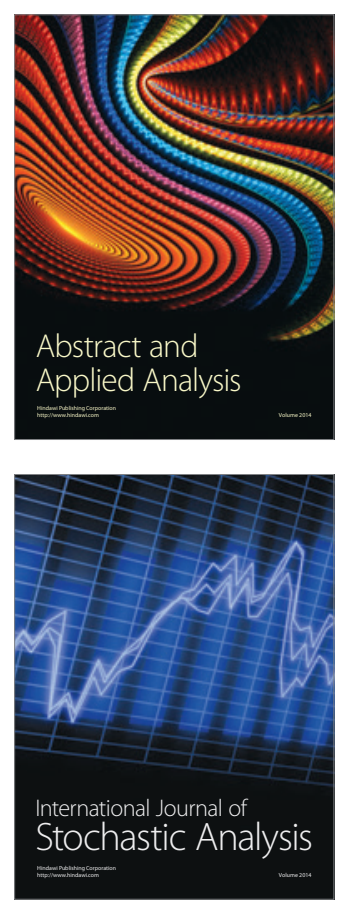

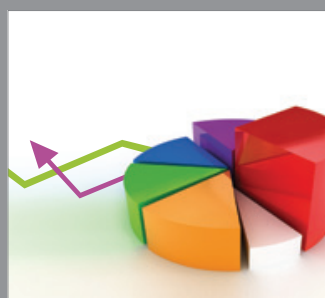

ournal of

Probability and Statistics

Promensencen
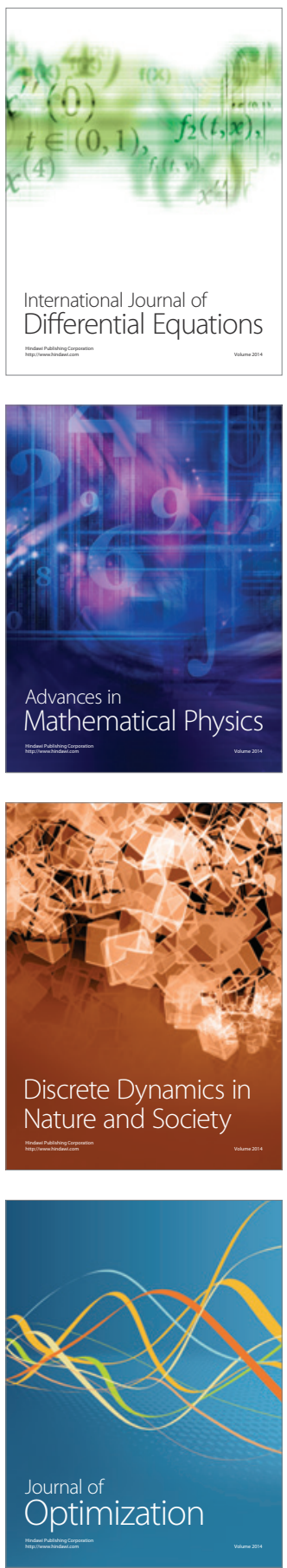